\title{
Salud mental en estudiantes de pregrado de la Facultad de Medicina de la Universidad Nacional Mayor de San Marcos Mental health in undergraduate students from Universidad Nacional Mayor de San Marcos School of Medicine
}

\author{
Alberto Perales ${ }^{1, a}$, Manuel Izaguirre ${ }^{1, \mathrm{~b}}$, Elard Sánchez $(\dagger)^{1, \mathrm{c}}$, Lorenzo Barahona ${ }^{1, \mathrm{~d}}$, Martha Martina ${ }^{1, \mathrm{e}}$, Isabel Amemiya ${ }^{1, \mathrm{f}}$, Ana Delgado ${ }^{1, \mathrm{~g}}$, \\ Imelda Domínguez ${ }^{1, \mathrm{~h}}$, Miguel Pinto, ${ }^{1, \mathrm{i}}$ Raúl Cuadros ${ }^{1, j}$, Alberto Padilla ${ }^{1, k}$ \\ ${ }^{1}$ Grupo de Investigación en Violencia y Salud, Instituto de Ética en Salud, Facultad de Medicina, Universidad Nacional Mayor de San Marcos. Lima, Perú. \\ a Médico psiquiatra, doctor en medicina, diplomado en ética. ORCID: https://orcid.org/0000-0002-3940-5182 \\ ${ }^{\mathrm{b}}$ Médico pediatra, doctor en educación. ORCID: https://orcid.org/0000-0002-0768-7402 \\ ${ }^{\circ}$ Médico psiquiatra, doctor en medicina \\ ${ }^{\mathrm{d}}$ Médico psiquiatra, doctor en medicina. ORCID: https://orcid.org/0000-0001-9885-0537 \\ e Licenciada en enfermería, doctora en ciencias de la salud. ORCID: https://orcid.org/0000-0002-8410-9120 \\ ${ }^{\dagger}$ Médico cirujano, magister en medicina. ORCID: https://orcid.org/0000-0002-5502-4785 \\ ${ }^{9}$ Médico cirujano, magister en nutrición, doctor en educación. ORCID: https://orcid.org/0000-0002-4507-1449 \\ ${ }^{\mathrm{h}}$ Médico especialista en enfermedades infecciosas y tropicales. ORCID: https://orcid.org/0000-0002-8270-9405 \\ 'Estudiante de medicina, ORCID: https://orcid.org/0000-0001-5789-3960 \\ 'Médico residente de psiquiatria infantil, ORCID: https://orcid.org/0000-0001-5196-1758 \\ ${ }^{k}$ Licenciado en estadística
}

An Fac med. 2019;80(4):443-50 / DOI: https://doi.org/10.15381/anales.v80i4.17142

Correspondencia:

Alberto Perales

perales.alberto@gmail.com

Recibido: 2 de diciembre 2019

Aceptado: 22 de diciembre 2019

Publicación en línea: 28 de diciembre 2019

Conflictos de interés: Los autores declaran no tener conflictos de interés.

Fuente de financiamiento: Universidad Nacional Mayor de San Marcos, con el código de estudio A17010351, mediante Resolución Rectoral No: 04274 - R - 17.

Contribuciones de autoría: AP lideró y realizó todas las actividades del estudio: planeamiento, ejecución (dirigió la encuesta), descripción, interpretación, y elaboración del artículo para publicación. MI participó en el planeamiento, ejecución (recolección de datos), descripción e interpretación. ES, LB y MM participaron en el planeamiento y ejecución (recolección de datos). IA participó en la revisión bibliográfica, planeamiento y ejecución (recolección de datos); además, aportó el instrumento de resiliencia. $A D, I D, M P$ y $R C$ participaron en la revisión bibliográfica, planeamiento y ejecución del estudio y recolección de datos. MP coordinó con los grupos federados estudiantiles. $R C$ coordinó la ejecución de la encuesta y A. Padilla fue el responsable del diseño de la muestra y del análisis estadístico. Todos los autores aprobaron la versión final del artículo.

Citar como: Perales A, Izaguirre M, Sánchez E, Barahona L, Martina M, Amemiya I, et al. Salud mental en estudiantes de pregrado de la Facultad de Medicina de la Universidad Nacional Mayor de San Marcos. An Fac med. 2019;80(4): 443-50. DOl: https:/doi. org/10.15381/anales.v80i4.17142
Resumen

Introducción. Estudios previos evidencian problemas de salud mental en estudiantes de pre-grado de la Facultad de Medicina de la Universidad Nacional Mayor de San Marcos (UNMSM). Objetivo. Evaluar si existen diferencias entre la frecuencia de problemas de salud mental entre los estudiantes de primer año, y el resto de años de estudio, en alumnos de las cinco escuelas profesionales de la Facultad de Medicina de la UNMSM. Métodos. Estudio transversal. Se utilizaron instrumentos validados para evaluar problemas de salud mental: intento suicida, pensamiento homicida, conducta disocial, problemas con el alcohol, depresión y angustia. Adicionalmente, se midieron niveles de resiliencia. Se incluyeron estudiantes de todas las escuelas profesionales de la Facultad de Medicina, en dos grupos de análisis: estudiantes de primer año y estudiantes de los otros años. Resultados. Se evidenció que en alumnos de primer año se encontró un $7,5 \%$ de intento suicida, $18,9 \%$ de conducta disocial y $21,9 \%$ de pensamiento homicida de prevalencia de vida. Asimismo, se encontró un $4,8 \%$ de problemas con el alcohol, $8,5 \%$ de depresión y 3,2\% de angustia de prevalencia periódica. La frecuencia de problemas de salud mental fue mayor según transcurrieron los años de estudio, aunque con diferencias estadisticamente significativas sólo en depresión, angustia y problemas con el alcohol. El 29,3\% de encuestados tuvo bajo nivel de resiliencia. Conclusión. Se encontraron frecuencias preocupantes de problemas de salud mental en los estudiantes de pregrado de la Facultad de Medicina de la UNMSM, aunque un porcentaje de alumnos ya ingresa a la universidad afectado por ellos. El entorno universitario sería factor agravante en depresión, ansiedad y problemas con el alcohol.

Palabras clave: Salud Mental; Facultades de Medicina; Educación de Pregrado en Medicina; Resiliencia Psicológica (fuente: DeCS BIREME).

\section{Abstract}

Introduction. Previous studies pointed out mental health problems in undergraduate health students of school of medicine from Universidad Nacional Mayor de San Marcos (UNMSM). Objective. To assess if there are differences between the frequency of mental health problems between first-year students and other years' students from five professional schools of the UNMSM school of medicine. Methods. A cross-sectional study. Validated instruments were used to assess mental health problems: suicidal intent, homicidal thinking, disocial behavior, alcoholism, depression and anguish. In addition, levels of resilience were evaluated. Students from all professional schools of the Faculty of Medicine were included in two analysis groups: first-year students and other years' students. Results. 7,5\% of suicide attempts, $18,9 \%$ of disocial behavior and $21,9 \%$ of homicidal thinking lifetime prevalence were found in first-year students. Similarly, 4,8\% of alcoholism, $8,5 \%$ of depression and $3,2 \%$ of anxiety point prevalence were found on same group of study. The frequency of mental health problems was higher according to the years of study, with statistically significant differences only in depression, anxiety and alcoholism. 29,3\% of respondents have a low level of resilience Conclusion. Concerning frequencies of mental health problems were found in undergraduate students of UNMSM School of Medicine, although a percentage of students were admitted in university with mental health problems. The university environment would be an aggravating factor in depression, anxiety and alcohol problems.

Keywords: Mental Health; Schools, Medical; Undergraduate Medical Education; Resilience, Psychological (source: MeSH NLM). 


\section{INTRODUCCIÓN}

La Organización Panamericana de la Salud ha precisado que los trastornos mentales, neurológicos específicos, debidos al consumo de sustancias, y el suicidio, constituyen causa importante de discapacidad y mortalidad. Suponen, además, que estos trastornos constituyen una tercera parte de todos los años perdidos por discapacidad (APD) y una quinta parte de todos los años de vida ajustados en función de la discapacidad (AVAD) en la región de las Américas, encontrándose en América del Sur, mayores proporciones de discapacidad debida a enfermedades mentales comunes ${ }^{(1)}$.

Desde el 2010, el grupo de investigación del Instituto de Ética en Salud, de la Facultad de Medicina de la Universidad Nacional Mayor de San Marcos (UNM$\mathrm{SM})$, viene estudiando los problemas de salud mental en estudiantes de las cinco Escuelas Académico Profesionales (EAPs) que la integran. En dicho año, se encontraron prevalencias de vida preocupantes de problemas de salud mental en la Escuela de Medicina: intento suicida (4\%), ideación homicida $(12,7 \%)$, indicadores de conducta antisocial $(19,2 \%)$ y de prevalencia puntual preocupante con el alcohol (7,3\%) ${ }^{(2)}$. En el mismo año, en alumnas de la Escuela de Enfermería se evidenciaron frecuencias de 10,2\% en intento suicida, 7,6\% de ideación homicida, $26,9 \%$ de indicadores de conducta antisocial y $4,2 \%$ de problemas con el alcohol (3). En el 2012, se ampliaron los estudios a las tres EAPs restantes: Obstetricia ${ }^{(4)}$, Nutrición ${ }^{(5)}$ y Tecnología Médica ${ }^{(6)}$; hallandose frecuencias de $8,3 \%, 9,4 \%$ y $7,6 \%$ de intento suicida; $6,9 \%, 13,9 \%$, y $11 \%$ de pensamiento homicida; $21,7 \%, 27,5 \%$ y $18,6 \%$ de indicadores de conducta disocial; y $3,1 \%, 5,2 \%$ y $4,7 \%$ de problemas con el alcohol, respectivamente.

Actualmente, la población estudiantil de las cinco EAPs de dicha facultad de medicina, (Medicina, Enfermería, Obstetricia, Nutrición y Tecnología Médica) alcanza un aproximado de 3000 alumnos. Anualmente ingresan alrededor de 500 estudiantes seleccionados en virtud de sus conocimientos; sin embargo, las variables de salud mental y desarrollo humano, quedan, en tal selección, bastante inciertas, a pesar del riesgo que por vulnerabilidad etaria afecta a este grupo adolescente y adulto joven ${ }^{(7,8)}$.

Los estudios previos señalados permiten considerar dos interrogantes: a) ¿Cuáles son las prevalencias actuales de los citados problemas de salud mental? $y$, b) ¿La salud mental de los estudiantes se afecta por influencia de la universidad o algunos de ellos inician sus estudios ya afectados?. Para dar respuesta a estas preguntas, la presente investigación tuvo como objetivo evaluar si existen diferencias entre la frecuencia de problemas de salud mental entre los estudiantes de primer año, y el resto de años de estudio, en alumnos de las cinco escuelas profesionales de la Facultad de Medicina de la UNMSM

\section{MÉTODOS}

\section{Diseño del estudio}

Se realizó un estudio observacional y transversal, para determinar la frecuencia de patologías de salud mental entre alumnos de primer año y de los años subsiguientes en estudiantes de pregrado de la Facultad de Medicina de la UNMSM.

\section{Población y muestra}

La población investigada fue la de estudiantes de pregrado de la Facultad de Medicina de la UNMSM, comprendida entre el primero y el cuarto año de estudios; debido a que los de quinto año, en cuatro de las escuelas, se distribuyen en el territorio nacional para realizar su internado, lo cual impidió su participación. De acuerdo a estas consideraciones, se calculó una población total de 2435 estudiantes, siendo la unidad de análisis el alumno de pregrado registrado como alumno en una de las cinco EAPs de la Facultad de Medicina de la UNMSM, ser de nacionalidad peruana, y haber aceptado el consentimiento informado.

El diseño de la muestra se hizo sobre los alumnos matriculados oficialmente (Tabla 1). Cada escuela profesional fue considerada un conglomerado y los años, estratos. Se formaron dos grupos de análisis: a) estudiantes de primer año, (aún sin influencia de la universidad) y evaluar si el alumno ingresaba con problemas de salud mental; $y, b$ ) estudiantes de los otros años, con influencia de la escuela profesional. En el grupo de primer año se entrevistó al universo completo. En el otro grupo se tomó una muestra probabilística de tipo complejo que comprendió estratificación y probabilidades desiguales de selección. Se obtuvo una muestra teórica de 1275 estudiantes. Se aceptó una tasa de no respuesta del $20 \%$ teniendo en cuenta que la participación de los estudiantes era voluntaria, por lo que, finalmente, se programó una muestra de 1700 estudiantes.

\section{Variables de estudio}

Las variables a estudiar fueron la presencia de problemas de salud mental: intento suicida, pensamiento homicida, conducta disocial, problemas con el alcohol, depresión y angustia, mediante instrumentos validados, cuya metodología se detalla en publicaciones previas ${ }^{(2-6)}$.

En esta investigación se agregó el uso de la Escala de Resiliencia de Wagnild y Young ${ }^{(9)}$, traducida al español y validada en el Perú ${ }^{(10)}$. Este instrumento estima los niveles de resiliencia en las siguientes dimensiones: ecuanimidad, sentirse bien solo, confianza en sí mismo, perseverancia, y satisfacción. Consta de 25 ítems, cada uno con un puntaje de 1 al 7, de acuerdo a una escala Likert. Se considera que los puntajes más altos son indicadores de mayor resiliencia, por lo que el rango de puntaje varía entre 25 y 175 puntos.

Adicionalmente, se recolectó información sobre variables que podrían actuar como posibles factores asociados como el sexo, escuela profesional (Medicina Humana, Enfermería, Obstetricia, Nutrición, Tecnología Médica), año de estudios, intentos de ingreso a la universidad, depresión, angustia, trabajo, con quien vive, o la condición de migrante a Lima Metropolitana.

Antes de aplicar los instrumentos, se dio una sesión explicativa a los alumnos. Los datos fueron recolectados entre octubre y noviembre de 2017, y los ejecutores fueron docentes investigadores de la Facultad de Medicina de la UNMSM. 
Tabla 1. Alumnos matriculados y distribución de entrevistados, por grupos de años de estudio, según escuela profesional. Facultad de Medicina, Universidad Nacional Mayor de San Marcos, 2017.

\begin{tabular}{|c|c|c|c|c|c|c|}
\hline \multirow{2}{*}{ Escuela profesional } & \multicolumn{3}{|c|}{ Alumnos matriculados } & \multicolumn{3}{|c|}{ Grupos de año de estudios } \\
\hline & Primero & Segundo a cuarto & Total & Primero & Segundo a cuarto & Total \\
\hline Medicina Humana & 186 & 553 & 739 & 141 & 376 & 517 \\
\hline Enfermería & 97 & 260 & 357 & 81 & 193 & 274 \\
\hline Obstetricia & 96 & 325 & 421 & 83 & 181 & 264 \\
\hline Nutrición & 61 & 211 & 272 & 61 & 128 & 189 \\
\hline Tecnología Médica & 166 & 480 & 646 & 102 & 224 & 326 \\
\hline Total & 606 & 1829 & 2435 & 468 & 1102 & 1570 \\
\hline
\end{tabular}

\section{Análisis estadístico}

La información se introdujo al sistema de gestión de bases de datos Microsoft Access $201{ }^{\circledR}$. Al concluir, se construyó una base de datos integrada en el programa IBM SPSS Statistics for Windows, version 21 (IBM Corp., Armonk, N.Y., USA. $2012{ }^{\circledR}$ ). Se realizó el análisis de consistencia y la depuración consiguiente.

Se aplicaron pruebas t y $\mathrm{F}$ ajustada para el análisis bivariado, y regresión logística para el análisis multivariado. Se realizó uno por cada problema de salud mental, en el que se incluyeron las variables consideradas como posibles factores, antes mencionadas. Asimismo, en forma adicional, se incluyó a otros problemas de salud mental, distintos al outcome de estudio.

Las medidas de asociación se reportan como Odds Ratio (OR) con sus respectivos intervalos de confianza al 95\%. Se consideró un valor de $\mathrm{p}<0,05$ para determinar diferencias significativas en todas las pruebas estadísticas.

\section{Aspectos éticos}

Todos los estudiantes entrevistados firmaron un consentimiento informado, el cual fue separado del cuestionario previo al análisis para mantener la confidencialidad de los datos. Al encuestado se le identificó por un código asignado en el gabinete, sin posibilidad de contacto ni identificación en la base de datos. El proyecto fue aprobado por el Comité de Investigación de la Facultad de Medicina UNMSM.

\section{RESULTADOS}

Se analizó la información de 1570 participantes, quienes tuvieron un prome- dio de edad de 21 años, con un 65\% de mujeres, y $78 \%$ de ellos nacidos en Lima Metropolitana. La mayoría de ellos (99\%) refirió ser soltero; el 14\%, trabajaba; y el $78 \%$ vivía con familia nuclear. Adicionalmente, el 31\% refirió que la ocupación predominante del jefe del hogar fue de categoría profesional.

Se encontró una frecuencia de intentos suicidas, durante toda la vida, de 8,5\%; mientras que en el último año y en los últimos seis meses, estas cifras fueron de $2 \%$ y $1,2 \%$ (Tabla 2). Al indagar sobre intentos suicidas en el último mes, se evidenció una frecuencia de $0,5 \%$. Al realizar un modelo multivariado, se encontró que el pertenecer a la EAP Enfermería (OR: 2,18; IC 95\% 1,08-4,41), el diagnostico de depresión (OR: 5,40; IC $95 \% 2,95-9,89$ ) y de angustia (OR: 2,56 ; IC 95\% 1,20-5,50), estuvieron asociados en forma estadísticamente significativa a intentos suicidas.

Respecto a los motivos del intento suicida; el $48 \%$ de los estudiantes que lo intentó, lo asoció a problemas o conflictos con sus padres o a la muerte de alguno de ellos; mientras que el 17,8\% lo vinculó a problemas con su salud o apariencia física. El 16,5\% lo relacionó a problemas con los estudios; y el $12 \%$ a conflicto o separación de pareja o de enamorado/a.

Del grupo afectado, el 46,2\% intentó suicidarse una sola vez, mientras que el $34,8 \%$ lo hizo en dos oportunidades o más. La modalidad más frecuente fue por medio de pastillas, en el 31,6\%; cortarse las venas, 23,1\%; y ahorcamiento, 19,2\%. Aunque otros procedimientos suicidas fueron utilizados con menor frecuencia, no deben subestimarse, pues su alta peli- grosidad no deja dudas sobre la intención letal de la motivación. Señalamos entre ellos: tomar substancias tóxicas, arrojarse desde una posición de altura, aventarse ante un vehículo en marcha, usar arma de fuego, entre otros.

A pesar de lo dramático que resulta un intento suicida en la existencia de una persona, el $46,4 \%$ de los estudiantes que lo intentaron, no consultó con nadie posteriormente; el 20,5\% acudió a un psicólogo y el $18,2 \%$ a un familiar o amigo no médico. Asimismo, el 16,2\% señaló haber considerado la posibilidad de repetir el intento.

Por otro lado, la presencia de pensamiento homicida alguna vez en la vida se encontró en un 22\% de los encuestados (Tabla 2). 7,7\% refirió haberlos tenido en el último año; $5,9 \%$, en los últimos seis meses y $3,4 \%$ durante el último mes. En el modelo multivariado, se encontró que el sexo masculino, el pertenecer a la EAP de Enfermería (OR: 1,93; IC 95\% 1,25 2,99), a la EAP de Nutrición (OR: 1,76; IC 95\% 1,05-2,94); o el tener diagnósticos de depresión (OR: 3,80; IC 95\% 2,406,00) o angustia (OR: 2,39; IC 95\% 1,25 $-4,57)$, estuvieron asociados a la presencia de pensamiento homicida. Sin embargo, el sexo femenino se mostró como un factor protector ante la presencia de este problema de salud mental (OR: 0,34; IC 95\% 0,24-0,49).

En cuanto a la conducta disocial, el $21 \%$ de los encuestados tuvo un reporte positivo (Tabla 2). Las conductas disociales más frecuentes fueron: estar en peleas que acabaron a golpes (37,9\%), mentir $(30,8 \%)$ y robar $(30,7 \%)$. En forma similar al pensamiento homicida, 
se encontró que el pertenecer a la EAP Nutrición (OR: 1,92; IC 95\% 1,10 - 3,34), y tener diagnóstico de depresión (OR: 2,02; IC 95\% 1,13 - 3,64) estuvieron asociados a la presencia de conducta disocial. Mientras que, ser del sexo femenino (OR: 0,14; IC 95\% 0,10-0,21) y trabajar al mismo tiempo que estudiar (OR: 0,66; IC 95\% 0,44-0,99), se presentaron como factores protectores.

Los problemas con el alcohol se presentaron en el 7,1\% (Tabla 2), de los estudiantes y se encontró como único factor asociado al sexo; siendo el sexo femenino, un factor protector ante esta conducta (OR: 0,24; IC 95\% 0,14-0,42).

El 11,7\% de los encuestados presentó depresión en la escala de Zung; y se evidenció que el sexo femenino (OR: 3,56; IC 95\% 1,93 - 6,54) y el diagnóstico de angustia (OR: 36,4; IC 95\% 18,89-70,14) fueron factores asociados a esta patología. Mientras que el 5,6\% presentó angustia en la escala de Zung; siendo la depresión el único factor asociado (OR: 38,32; IC 95\% 19,21 - 76,41)

El 29,3\% de los estudiantes presentó nivel bajo de resiliencia (Tabla 2); sólo $9 \%$ puntuó en nivel alto y el $61,7 \%$ estuvo en nivel intermedio. El análisis multivariado de regresión logística encontró menor riesgo significativo en la EAP de Tecnolo- gía Médica (OR: 0,52; IC 95\% 0,29-0,94) y mayor riesgo para depresión (OR: 6,15; IC 95\% 3,76-10,06).

Al evaluar las diferencias en los problemas de salud mental entre estudiantes de primer año y de otros años, se encontraron diferencias significativas en depresión, angustia y problemas con el alcohol (Tabla 2).

\section{DISCUSIÓN}

El valor de un trabajo de investigación depende no sólo de sus resultados específicos sino, también, de las hipótesis de investigación que genera. Los resultados presentados evidencian problemas de salud mental en la población estudiada.

De la comparación entre los resultados de alumnos de primer año y de los "otros años" se desprenden observaciones importantes. En primer lugar, que un porcentaje de estudiantes llega a la universidad ya afectado en su salud mental, pues los de primer año ya muestran las siguientes frecuencias: intento suicida, 7,5\%; conducta disocial, $18,9 \%$; y pensamiento homicida $21,9 \%$; mientras que mencionan problemas con el alcohol, 4,8 $\%$; depresión, 8,5 \%; y angustia, 3,2\%. En segundo lugar, la comparación con los años superiores refleja una gradiente diferencial, siempre favorable a "los otros años", con diferencias significativas sólo en depresión, (4,2\%); angustia (3,2\%) y problemas con el alcohol (3\%).

Una hipótesis plausible a propósito de tales resultados, apuntaría a que el contexto universitario no participaría en la causalidad de las patologías detectadas, pero sí como factor agravante. En las conductas cuyas prevalencias no muestran diferencias significativas, (intento suicida, pensamiento homicida, conducta disocial) la influencia del ambiente académico parece ser menor; mientras que, en aquellas que si muestran diferencia: depresión, angustia y problemas con el alcohol, la influencia del entorno universitario sería mayor. Respecto a la resiliencia, se observó que esta es mayor en "los otros años" $(1,6 \%)$; por lo que cabría la hipótesis de que, si esta aumente con la experiencia de la vida universitaria, podría configurarse en factor protector ante la aparición de problemas de salud mental.

Desde otro ángulo, cabe examinar cada problema de salud mental en forma individual, aunque recordando que la Organización Mundial de la Salud (OMS), resalta la importancia del manejo integral de estos problemas en la edad adolescente y adulto joven ${ }^{(8)}$.

Tabla 2. Diferencias en los problemas de salud mental entre estudiantes de primer año y otros años, de la Facultad de Medicina de la Universidad Nacional Mayor de San Marcos, 2017.

\begin{tabular}{|c|c|c|c|c|}
\hline Problema de salud mental & Grupo de Estudio & $\mathbf{N}$ & $\%$ (IC 95\%) & Valor $\mathrm{p}^{*}$ \\
\hline \multirow{2}{*}{ Intento suicida } & Primer año & 465 & $7,5(5,0-9,9)$ & \multirow{2}{*}{0,431} \\
\hline & "Otros años" & 1081 & $8,7(6,9-10,5)$ & \\
\hline \multirow{2}{*}{ Pensamiento homicida } & Primer año & 454 & $21,9(18,0-25,7)$ & \multirow{2}{*}{0,937} \\
\hline & "Otros años" & 956 & $22,1(19,2-24,9)$ & \\
\hline \multirow{2}{*}{ Depresión } & Primer año & 457 & $8,5(5,9-11,0)$ & \multirow{2}{*}{$0,018^{*}$} \\
\hline & "Otros años" & 1077 & $12,7(10,6-14,8)$ & \\
\hline \multirow{2}{*}{ Angustia } & Primer año & 461 & $3,2(1,5-4,9)$ & \multirow{2}{*}{$0,016^{*}$} \\
\hline & "Otros años" & 1076 & $6,4(4,8-7,9)$ & \\
\hline \multirow{2}{*}{ Conducta disocial } & Primer año & 461 & $18,9(15,3-22,5)$ & \multirow{2}{*}{0,228} \\
\hline & "Otros años" & 1080 & $21,7(19,1-24,3)$ & \\
\hline \multirow{2}{*}{ Problemas con alcohol } & Primer año & 462 & $4,8(2,8-6,8)$ & \multirow{2}{*}{$0,035^{*}$} \\
\hline & "Otros años" & 1085 & $7,8(6,1-9,6)$ & \\
\hline \multirow{2}{*}{ Resiliencia } & Primer año & 448 & $12,7(9,6-15,7)$ & \multirow{2}{*}{0,410} \\
\hline & "Otros años" & 1059 & $14,3(12,0-16,5)$ & \\
\hline
\end{tabular}

$\left({ }^{*}\right)$ Se consideró estadísticamente significativo un valor $\mathrm{p}<0,05$. 
La conducta suicida constituye una compleja manifestación humana, de difícil comprensión, especialmente cuando ocurre en estudiantes universitarios a quienes asumimos disfrutando de la plenitud de su vitalidad juvenil. Más aún, si luego de superar la difícil valla del ingreso a estudios superiores, se hallan en la antepuerta del éxito profesional y social. ¿Qué puede ocurrir en la mente de estos jóvenes que, en lugar de un proyecto de vida se plantean uno de muerte?. Estadísticamente, se señala que el suicidio es la tercera causa de muerte en el grupo etario de 15 a 24 años ${ }^{(11)}$; y es la tercera causa de muerte en población etaria universitaria de Estados Unidos. La primera y segunda causas de muerte en este grupo poblacional: daño no intencional y homicidio, pueden también vincularse a ideación suicida ${ }^{12)}$, cuyo incremento, de acuerdo a un reciente meta-análisis, comienza, para los estudiantes de medicina, en la escuela respectiva ${ }^{(13)}$.

La opinión experta señala que la causalidad de la conducta suicida es multifactorial; y depende, como evidencia la investigación científica, de factores internos-únicos y exclusivos de la persona- y externos, derivados de múltiples situaciones y circunstancias [generadoras de estrés] ${ }^{(14)}$. Más aún, en investigaciones anteriores, ya señaladas ${ }^{(2-6)}$, se ha descrito que esta conducta escalona una dinámica dimensional, de usual inicio a temprana edad, generando una gradiente de comportamientos que van, desde deseos de morir, ideación suicida, plan suicida, intento suicida, hasta, finalmente, el suicidio como objetivo final del propósito autodestructivo. En tal perspectiva, el caminar por la senda suicida, desde su inicio hasta el éxito letal, puede durar horas, años, o nunca completarse. En esta secuencia, la realidad clínica demuestra que tal potencial autodestructivo puede permanecer latente en el estudiante $y$ manifestarse, tiempo después, en la vida profesional. Ello contribuiría a explicar, según Joy Albuquerque y Sarah Tulk, por qué los médicos, comparados con los no médicos, tienen un riesgo mayor de morir por suicidio: en varones (OR: 1,41; IC 95\%: 1,21-1,65); y, mayor aún, en mujeres (OR: 2,27; IC 95\%: 1,90-2,73) ${ }^{(13)}$.
Incrementa tal riesgo que los médicos y profesionales de salud dispongan, por circunstancias laborales, de mayor acceso a medios suicidas.

Igual preocupación existe entre enfermeras. Mientras que algunos investigadores señalan que nadie sabe, a ciencia cierta, cuáles son las cifras reales sobre el tema ${ }^{(14)}$ otros reportan cifras preocupantes. Tal es el caso de Judy Davidson y colaboradores, quienes informaron, en Estados Unidos, que la primera investigación a nivel nacional demostró porcentajes mayores de suicidio en estos profesionales comparados con los no enfermeros. La incidencia fue de 12/100 000 personas-año en el sexo femenino, y 39,8 por 100000 en el masculino, cifras significativamente mayores a las observadas en ambos sexos de población general (7,6 y 28,2 por 100000 persona-año, respectivamente, $p<0,001)^{(15)}$.

El intento suicida evidenció un riesgo mayor si el alumno presentaba depresión (OR: 5,40; IC 95\% 2,95 - 9,89); si presentaba angustia (OR: 2,56; IC 95\% 1,20 $-5,50)$ o si pertenecía a la escuela de enfermería (OR: 2,18; IC 95\% 1,08-4,41). En la literatura internacional, la presencia de depresión y ansiedad como factores asociados a la conducta suicida ha sido ampliamente reconocida ${ }^{(16)}$. El hecho de que el riesgo de intento suicida tenga significación estadística sólo en la escuela de enfermería, permite, considerando la teoría de vulnerabilidad-estrés, ya mencionada y aplicada a la conducta suicida, hipotetizar que tal escuela se estaría convirtiendo en estresor institucional, lo que requiere ser estudiado con mayor detenimiento.

En relación al pensamiento homicida, debemos puntualizar que se trata de un término médico referido la presencia de ideas con tal intención. En una perspectiva dimensional, el concepto cubre un amplio espectro de manifestaciones que van desde simples fantasías o pensamientos, usualmente revanchistas o vengativos, hasta planes muy elaborados con el propósito de llevarlos a la acción. Venimos estudiándolo porque la teoría psicoanalítica lo vincula a la dinámica etiológica del suicidio ${ }^{(17)}$. Freud conside- raba al suicidio como agresión homicida volcada hacia sí mismo, dirigida contra un objeto de amor introyectado con catexis ambivalente ${ }^{(18)}$. La relación de estas dos variables (conducta suicida y pensamiento homicida) alcanzó evidencia estadísticamente significativa en uno de nuestros estudios previos ${ }^{(2)}$. Esta asociación es también reportada internacionalmente ${ }^{(19)}$. En nuestro país, con cierta frecuencia, el periodismo destaca tal asociación en casos de feminicidio; quienes describen, no infrecuentemente, que el feminicida se suicida luego de asesinar a su pareja y, a veces, a sus hijos.

En nuestro estudio, los resultados asocian al pensamiento homicida con un riesgo mayor si el estudiante pertenecía a la escuela de enfermería (OR: 1,93; IC $95 \% 1,25-2,99)$; así como, si pertenecía a la escuela de nutrición (OR: 1,76; IC 95\% 1,05 - 2.94). Del mismo modo, el riesgo aumentaba si presentaba depresión (OR: 3,80; IC 95\% 2,40-6,00) o angustia (OR: 2,39; IC 95\%: 1,25 - 4,57); pero disminuía, si el estudiante era de sexo femenino (OR: 0,34; IC 95\% 0,24 $0,49)$. Mats Persson y colaboradores, en un estudio comparativo entre acusados de actos violentos y pacientes psiquiátricos, no encontraron mayores diferencias en las prevalencias. En cuanto a la concordancia entre pensamiento violento y acto resultante, señalaron que se observó a nivel grupal pero que, a nivel individual, deben considerarse factores adicionales del entorno para evaluar el riesgo ${ }^{(20)}$. Por tal consideración, debemos volver a preguntarnos qué papel juegan el clima organizacional de las escuelas de enfermería y de nutrición, que aparecen asociadas en forma estadísticamente significativa a pensamiento homicida, además de los factores de depresión y angustia para favorecer esta conducta.

Otros autores, van aún más allá de una simple asociación, y consideran que las conductas, suicida y homicida, pertenecen a un continuo conductual de riesgo que conviene analizar, prevenir y tratar, no individualmente sino en conjunto. Barrios y colaboradores, usando los datos del National College Health Risk Behavior Survey, encontraron que aquellos estudiantes que habían reportado ideación suicida, mostraban, también, mayor fre- 
cuencia de otras conductas de riesgo, tales como: portar armas, involucrarse en peleas físicas, nadar o manejar después de ingerir alcohol, y usar, pocas veces o nunca, el cinturón de seguridad ${ }^{(12)}$.

Susan Harter y colaboradores estudiaron un modelo de ideación suicida y homicida, encontrando ambas variables altamente correlacionadas $(r=0,55)$. La percepción de competencia y adecuación, y el soporte social, brindado por pares y padres, predijeron dos mediadores en el modelo, uno de adaptación/ depresión, compuesto por (autoestima, esperanza/desesperanza y afecto alegría/ depresión) y otra, caracterizada por agresión física inducida por cólera. Estas, a su vez, predijeron la presencia de pensamiento suicida y de violencia; por lo que se postula, que los pensamientos de violencia dirigidos hacia otros (homicida) o a sí mismos (suicidio) se entrelazan en una misma dinámica y origen psicosocial (21).

En cuanto a la depresión y la angustia, clásicamente han sido asociadas a conducta suicida, problemas de rendimiento académico, problemas con el alcohol y otras dificultades de los estudiantes de pregrado. Los resultados encontrados en nuestros estudiantes son concordantes con los reportados en la literatura internacional y nacional ${ }^{(22-25)}$. Asimismo, los factores que se asociaron estadísticamente a depresión fueron: sexo femenino (OR: 3,56 ; IC 95\% 1,93 - 6,54) y angustia (OR: 36,4; IC 95\% 18,89-70,14); mientras que el único factor asociado a angustia fue depresión (OR: 38,32; IC 95\% 19,21 - 76,41). Podemos deducir de ello que tales factores, depresión y angustia, parecen reforzarse uno al otro. En este sentido, Shawaz Iqbal y colaboradores destacaron el importante rol del estrés en ambos trastornos, pues, los estudios universitarios constituyen importante cambio existencial para los discentes, incluyendo un nuevo estilo de vida, nuevas amistades, nuevos retos y nuevas responsabilidades ${ }^{(26)}$. A ello se agrega, en muchos casos, la presión familiar que experimenta el estudiante para que logre el éxito anhelado, que se incrementa si la familia lo percibe como el trampolín humano para el cambio social y posible éxito económico. Por ello, Wanda Chernomas y Carla Shapiro enfatizaron la necesidad de que el cuerpo docente muestre la necesaria sensibilidad para detectar a tiempo las manifestaciones iniciales de estos cuadros en los estudiantes ${ }^{(27)}$.

Por su parte, las conductas disociales, requieren particular atención, especialmente por las carreras de salud que siguen los estudiantes de las cinco escuelas de la Facultad de Medicina UNMSM (28). La dinámica y causalidad de este problema exige estudios de mayor precisión, y su presencia y relevancia son indiscutibles. Los factores asociados fueron ser varón y trabajar al mismo tiempo que se estudia. Más aún, el riesgo aumenta si el estudiante pertenecía a la escuela de nutrición (OR: 1,92; IC 95\% 1,10 - 3,34) o si presentaba depresión (OR: 2,02 (IC 95\% 1,13 - 3,64). El problema de conductas disociales en estudiantes de ciencias de la salud es particularmente preocupante pues, ya como profesionales, habrán de asumir responsabilidad frente a vidas humanas. Tal como hemos planteado en otro trabajo, somos conscientes que el total de estudiantes de la Facultad de Medicina UNMSM requiere ser formado en ética; pero mientras la gran mayoría, sin problemas serios de salud mental podría beneficiarse con los métodos didácticos regulares, un grupo menor, con conductas disociales requerirá de técnicas innovadoras de enseñanza que refuercen su desarrollo moral ${ }^{(29)}$.

El único factor asociado a problemas con el alcohol fue ser varón (OR: 0,24; IC $95 \% 0,14-0,42$ ) que en nuestro país tiene un fuerte peso cultural. Los resultados de estudios previos sobre esta problemática en nuestros estudiantes señalan la importancia de actuar a tiempo. Conocemos que, en su dinámica, los adolescentes la utilizan como vía de descarga de múltiples tensiones, con el riesgo de habituación y dependencia al alcohol, pero también existen factores que la facilitan como depresión (30) y hábitos culturales que podrían ser manejados con intervenciones apropiadas. Estudios anteriores, ya citados, señalan que el problema se presenta con mayor intensidad en las escuelas de medicina (2) y de nutrición (5) con lo cual, una vez más, destaca la importancia de averiguar en qué medida, el entorno institucional contribuye al problema citado.
En cuanto a la resiliencia, nuestros resultados apuntan a un porcentaje importante de estudiantes con resiliencia baja $(29,3 \%)$ con riesgo estadísticamente significativo a presencia de depresión (OR: 6,15; IC 95\% 3,76-10,06). Hipotéticamente, un nivel bajo de resiliencia disminuiría las defensas del estudiante frente a los riesgos descritos ${ }^{(31)}$. Ello sugiere que un grupo de estudiantes ingresa a la universidad ya con este problema, aunque se aprecia que el nivel de resiliencia es mayor en el curso del tiempo. Conviene investigar qué tipo de experiencias y otros factores contribuyen a este aumento pues, de saber cómo incrementarla, la ayuda sería colectivamente positiva pues puede constituirse como un factor protector ante múltiples problemas de salud mental (32).

Finalmente, muchos otros autores han señalado la urgente necesidad de instalar programas de ayuda para los estudiantes universitarios con problemas de salud mental. Schwartz, destaca los buenos resultados de los centros de consejería psicológica en los campus universitarios que logran reducir el riesgo de suicidio en el alumnado ${ }^{(33)}$. Tales experiencias han demostrado la utilidad de los programas de intervención, aunque no exista consenso sobre cuál es la mejor modalidad ${ }^{(34)}$.

En resumen, los resultados expuestos expuestos señalan que un porcentaje de alumnos ingresa a la universidad ya con problemas de salud mental. A pesar de tales condiciones adversas, debe considerarse que tales jóvenes han alcanzado, con esfuerzo, acceder a estudios superiores, logrando un avance substancial en sus proyectos de vida. Merecen, por tanto, todo el apoyo de la universidad para coronar con éxito tal batalla existencial, por lo que cualquier intento de eliminarlos del ingreso debido a su patología sería la negación de toda posibilidad de superarla. Sin embargo, tales alumnos, de no ser identificados y ayudados oportunamente durante su periodo estudiantil, asumirían mayor riesgo, no sólo de afectar su rendimiento académico, sino que, cuando ya profesionales, aumentar la probabilidad de dañar a la sociedad y/o a sí mismos con conductas inadecuadas. 
Desde esta óptica, la situación descrita compromete la "responsabilidad social de la universidad". Este concepto se forja durante la segunda mitad del siglo $X X$, tanto por el impacto de las empresas sobre la sociedad cuanto por fallas en su gestión de modo ético y sostenible. Aplicado a la universidad resalta su compromiso con la sociedad en la cual existe, lo opuesto a un modelo multinacional del conocimiento masivo desarraigado de su responsabilidad local (35). Tal visión ha sido compartida por la Conferencia Mundial de Educación Superior, (UNESCO, 2009), al señalar: "Las instituciones de educación superior deben brindar a los estudiantes la posibilidad de desarrollar plenamente sus propias capacidades con sentido de responsabilidad social" (36).

En otras palabras, aunque el principal objetivo declarado de la universidad sea formar profesionales idóneamente capacitados, el Decano de la Facultad de Medicina UNMSM, Dr. Gerardo Ronceros comentó en la Conferencia Internacional sobre Ética en Salud: "Ética y Responsabilidad Social en Salud", realizada el 09 y 10 de Noviembre de 2018, en Lima, Perú: "actualmente se viene experimentando una transformación en el campo educativo, y se ha dejado atrás la creencia de que el único papel de la universidad consiste en la transferencia de conocimiento... por tanto, sus actividades deben demostrar que están más vinculadas al servicio del ser humano, de su realización personal, progreso ético, desarrollo profesional y vinculación solidaria con los intereses colectivos".

Así, la universidad no puede ni debe eludir tal compromiso con sus estudiantes y su país. Debiera, como señala Juan Enrique Mezzich, apoyarse en el enfoque de educación médica centrada en la persona ${ }^{(37)}$. Y, de ser así, focalizar su acción en el estudiante como ser humano incluyendo como objetivo institucional, el formar al estudiante no sólo como buen profesional sino ayudarlo a desarrollarse como persona cabal. Investigar cómo ayudar al estudiante universitario a enfrentar los problemas descritos, y apoyarlo en la realización de su proyecto de vida, ofreciéndole oportunidades y espacios académicos estructurados para el florecimiento de sus potencialidades personales, es obligación científica y responsabilidad moral de la universidad peruana del siglo XXI. Y por extensión, de los profesores sanmarquinos obligados a compartirlas.

Pese a que los problemas descritos evidencian fundadas sospechas de patología de salud mental en la población universitaria estudiada, creemos que el tamaño de la muestra pudiera haber sido insuficiente para validar otras diferencias significativas en las prevalencias comparadas entre años de estudio. Convendría tomar en cuenta este factor para próximos estudios.

Se concluye que se encontró frecuencias preocupantes de problemas de salud mental en los estudiantes de pregrado de la Facultad de Medicina de la UNMSM, y que un porcentaje de alumnos ingresó a la universidad ya con problemas de salud mental. El entorno universitario sería factor agravante en depresión, ansiedad y problemas con el alcohol

\section{AGRADECIMIENTOS}

Al Director de la Escuela de Medicina Humana, Dr. Roberto Shimabuku; A la Directora de la Escuela de Enfermería, Dra. María Arcaya; A la Directora de la Escuela de Obstetricia, Mg. Clara Díaz; A la Directora de la Escuela de Nutrición, Mg. Doris Delgado; y al Director de la Escuela de Tecnología Médica, Mg. José Antonio Paredes, por las facilidades prestadas para el desarrollo de la presente investigación. Y a nuestros alumnos de las cinco escuelas, objetivo central de nuestro interés moral y científico, por su voluntaria participación.

\section{REFERENCIAS BIBLIOGRÁFICAS}

1. Organización Panamericana de la Salud. La carga de los trastornos mentales en la Región de las Américas. Washington: OPS; 2018.

2. Perales A, Alarcón J, Sánchez E, Arcaya M, Cortez E, Parhuana A, et al. Conducta suicida en estudiantes de medicina de una universidad nacional peruana. En: Bustamante I, Rivera M, Matos L, Editores. Violencia y Trauma en el Perú. Desafíos y Respuestas. Lima: Mc Graw-Hill; 2013. (Programa Trauma y Salud Global)

3. Perales A, Alarcón J, Sánchez E, Arcaya M, Parhuana A, Caballero J, et al. Conducta Suicida en estu- diantes de Enfermería de la Facultad de Medicina de la Universidad Nacional Mayor de San Marcos. Informe Final. Proyecto 2010. Vicerrectorado de Investigación de la UNMSM; 2010.

4. Perales A, Sánchez E, Rosas M, Parhuana A, Parhuana A, Carrera R, et al. Conducta suicida en estudiantes de la escuela de Obstetricia de una universidad pública del Perú. Rev Peruana de Psiquiatria. 2014;4(1):5-9.

5. Perales A, Sánchez E, Parhuana A, Carrera R, Torres $\mathrm{H}$. Conducta suicida en estudiantes de la escuela de nutrición de una universidad pública peruana. Revista de Neuro-Psiquiatria. Rev de Neuro Psiquiatria. 2013;76(4):231-5

6. Perales A, Sánchez E, Arcaya M, Cortez E, Parhuana A, Carrera R, et al. Conducta suicida en estudiantes de la escuela de Tecnología Médica de una universidad pública peruana. El Antoniano. 2015;(126):11-5.

7. Organización Mundial de la Salud. [On line]. 2019. Suicidio [citado 24 setiembre 2019]. Disponible en: https://www.who.int/es/news-room/fact-sheets/ detail/suicide

8. Organización Mundial de la Salud. [On line]. 2019. Salud mental del adolescente [citado 24 setiembre 2019]. Disponible en: https://www.who.int/es/newsroom/fact-sheets/detail/adolescent-mental-health

9. Wagnild GM, Young HM. Development and psychometric evaluation of the Resilience Scale. J Nurs Meas. 1993;1(2):165-78.

10. Flores Crispin MS. Resiliencia y proyecto de vida en estudiantes del tercer año de secundaria de la UGEL 03. [Tesis para optar el grado de magister en psicología]. Lima: Universidad Nacional Mayor de San Marcos; 2008.

11. Arria AM, O'Grady KE, Caldeira KM, Vincent $K B$, Wilcox HC, Wish ED. Suicide ideation among college students: a multivariate analysis. Arch Suicide Res. 2009;13(3):230-46. DOI: 10.1080/13811110903044351

12. Barrios LC, Everett SA, Simon TR, Brener ND. Suicide ideation among US college students. Associations with other injury risk behaviors. J Am Coll Health. 2000;48(5):229-33. DOI: 10.1080/07448480009599309

13. Albuquerque J, Tulk S. Physician suicide. CMAJ. 2019;191(18):E505-E505. DOI: 10.1503/ cmaj. 181687

14. Sofer D. Suicide Among Nurses. AJN The American Journal of Nursing. 2018;118(8):14. DOI: 10.1097/01.NAJ.0000544147.83703.35

15. Davidson JE, Proudfoot J, Lee K, Zisook S. Nurse suicide in the United States: Analysis of the Center for Disease Control 2014 National Violent Death Reporting System dataset. Archives of Psychiatric Nursing. 2019;33(5):16-21. DOI: 10.1016/j. apnu.2019.04.006

16. Bedoya Cardona EY, Montaño Villalba LE. Suicidio y trastorno mental. CES Psicologia. 2016;9(2):179-201.

17. Freud S. La aflicción y la melancolía. Dans: Obras Completas. Madrid: Editorial Biblioteca Nueva; 1948. p. 1092.

18. Alec R. Chapter 30.1. Psychiatric emergencies. Dans: Kaplan H, Sadock B. Comprehensive Textbook of Psychiatry/VI. Baltimore: Williams \& Wilkins; 1995. p. 1740-1.

19. Hillbrand M. Homicide-suicide and other forms of co-occurring aggression against self and against others. Professional Psychology: Research and Practice. 2001;32(6):626-35. DOI: 10.1037/07357028.32.6.626 
20. Persson M, Sturup J, Belfrage $H$, Kristiansson M. Self-reported violent ideation and its link to interpersonal violence among offenders with mental disorders and general psychiatric patients. Psychiatry Res. 2018;261:197-203. DOI: 10.1016/j. psychres.2017.12.079

21. Harter S, Low SM, Whitesell NR. What Have We Learned from Columbine:The Impact of the SelfSystem on Suicidaland Violent Ideation Among Adolescents. Journal of School Violence. 2003;2(3):3-26.

22. Coffin Cabrera N, Álvarez Zúñiga M, Marín Coria A. Depresión e ideación suicida en estudiantes de la FESI: Un estudio piloto. Revista Electrónica de Psicología Iztacala. 2011;14(4):341-53.

23. Amézquita Medina ME, González Pérez RE, Zuluaga Mejía D. Prevalencia de la depresión, ansiedad y comportamiento suicida en la población estudiantil de pregrado de la Universidad de Caldas, año 2000. Revista Colombiana de Psiquiatria. 2003;32(4):341-56.

24. Arrivillaga Quintero M, Cortés Garcia C, Goicochea Jiménez VL, Lozano Ortiz TM. Caracterización de la depresión en jóvenes universitarios. Universitas Psychologica. 2004;3(1):17-26.

25. Saavedra Castillo J, Paz V, Vargas H, Nizama M, Vásquez F, Huamán J, et al. Estudio epidemiológico Metropolitano en Salud Mental 2002. Informe General. Anales de Salud Mental. 2002;18(1-2):61-127.

26. Iqbal S, Gupta S, Venkatarao E. Stress, anxiety and depression among medical undergraduate students and their socio-demographic correlates. Indian J Med Res. 2015;141(3):354-7. DOI: 10.4103/09715916.156571

27. Chernomas WM, Shapiro C. Stress, depression, and anxiety among undergraduate nursing students. Int J Nurs Educ Scholarsh. 2013;10. DOI: 10.1515/ ijnes-2012-0032

28. Perales A, Sánchez E, Barahona L, Oliveros M, Bravo E, Mendoza A, et al. Conducta y personalidad disociales en estudiantes de medicina: implicancias para la educación médica peruana. Anales de la Facultad de Medicina. 2018;79(3):238-43. DOI: 10.15381/anales.v79i3.15018

29. Perales A. Formación ética como soporte del desarrollo moral del alumno de carreras de ciencias de la salud: hacia una enseñanza centrada en las necesidades del estudiante como persona. Revista Peruana de Medicina Experimental y Salud Publica. 2019;36(1):100-5. DOI: 10.17843/ rpmesp.2019.361.4314

30. Valle R, Sánchez E, Perales A. Sintomatología depresiva y problemas relacionados al consumo de alcohol durante la formación académica de estudiantes de medicina. Revista Peruana de Medicina Experimental y Salud Publica. 2013;30(1):54-7.

31. Vinaccia S, Quiceno JM, Pedro EMS. Resiliencia en adolescentes. Revista Colombiana de Psicologia. 2007;16(0):139-46
32. Márquez M. La Resiliencia como factor de protección en Salud Mental en adolescentes de una Unidad Educativa de Valencia-Estado de Carabobo. [Tesis de Especialista en Salud y Desarrollo de Adolescentes]. Carabobo: Universidad de Carabobo; 2017.

33. Schwartz AJ. College student suicide in the United States: 1990-1991 through 2003-2004. J Am Coll Health. 2006;54(6):341-52. DOI: 10.3200/ JACH.54.6.341-352

34. Westefeld JS, Button C, Jr JTH, Kettmann JJ, Macconnell J, Sandil R, et al. College Student Suicide: A Call To Action. Death Studies. 2006;30(10):931-56. DOI: 10.1080/07481180600887130

35. Vallaeys F. La responsabilidad social universitaria: un nuevo modelo universitario contra la mercantilización. Revista iberoamericana de educación superior. 2014;5(12):105-17.

36. United Nations Educational, Scientific and Cultura Organization (UNESCO). Conferencia Mundial sobre la Educación Superior en el siglo XXI: Visión y acción, Paris, 1998. [On line]. UNESCO; 1998 [citado 25 setiembre 2019].

37. Mezzich JE, Perales A. Atención clínica centrada en la persona: principios y estrategias. Revista Peruana de Medicina Experimental y Salud Publica. 2016;33(4):794-800. DOI: 10.17843/rpmesp.2016.334.2567 